\title{
Substance $P$ antagonist improves both obesity and asthma in a mouse model
}

\author{
R. Ramalho ${ }^{1}$, J. Almeida ${ }^{2}$, M. Beltrão ${ }^{1}$, A. Pirraco ${ }^{3}$, R. Costa ${ }^{3}$, O. Sokhatska ${ }^{1}$, \\ L. Guardão ${ }^{4}$, C. Palmares ${ }^{1}$, J. T. Guimarães ${ }^{3,5}$, L. Delgado ${ }^{1,5}{ }^{,}$A. Moreira ${ }^{1,6}$ \& R. Soares ${ }^{3}$ \\ ${ }^{1}$ Immunology, Faculty of Medicine, University of Porto; ${ }^{2}$ Ciências Químicas e das Biomoléculas, Escola Superior de Tecnologia da Saúde do \\ Porto, Instituto Politécnico do Porto; ${ }^{3}$ Biochemistry (U38-FCT), Faculty of Medicine, University of Porto; ${ }^{4}$ Animal House Unit, Faculty of \\ Medicine, University of Porto; ${ }^{5}$ Clinical Pathology, Centro Hospitalar São João; ${ }^{6}$ Immuno-Allergology, Centro Hospitalar São João, Porto, \\ Portugal
}

To cite this article: Ramalho R, Almeida J, Beltrão M, Pirraco A, Costa R, Sokhatska O, Guardão L, Palmares C, Guimarães JT, Delgado L, Moreira A, Soares R. Substance P antagonist improves both obesity and asthma in a mouse model. Allergy 2013; 68: 48-54.

\section{Keywords}

animal model; asthma; NK1-R antagonist; obesity; substance $P$; treatment.

\section{Correspondence}

Renata Ramalho, Department of

Immunology, Faculty of Medicine, University

of Porto, Alameda Professor Hernâni

Monteiro, 4200 Porto, Portugal.

Tel.: +225513657

E-mail: rrenata@med.up.pt

Accepted for publication 20 September 2012

DOI:10.1111/all.12052

Edited by: Hans-Uwe Simon

\begin{abstract}
Background: Evidence suggests a causal relationship between obesity and asthma; however, the underlying mechanisms remain unknown. Substance P (SP), involved in neurogenic inflammation by acting through its receptor NK1-R, seems to participate in obese-asthma phenotype in mice.

Objectives: To evaluate the effect of a selective substance $\mathrm{P}$ receptor antagonist on a mouse model of diet-induced obesity and asthma.

Methods: Diet-induced obese Balb/c mice were sensitized and challenged with ovalbumin (OVA) and treated with a selective NK1-R antagonist or placebo. Serum glucose, insulin, IL-6, resistin, and OVA-specific IgE levels were quantified. A score for peribronchial inflammation in lung histology was used. Cells were counted in bronchoalveolar lavage fluid. Adipocyte sizes were measured.

Results: Ovalbumin-obese mice treated with NK1-R antagonist had lower weight $(P=0.0002)$, reduced daily food intake $(P=0.0021)$, reduced daily energy intake $(P=0.0021)$, reduced surface adipocyte areas $(P<0.0001)$, lower serum glucose $(P=0.04)$, lower serum insulin $(P=0.03)$, lower serum IL- $(P=0.0022)$, lower serum resistin $(P=0.0043)$, lower serum OVA-specific $\operatorname{IgE}(P=0.035)$, and lower peribronchial inflammation score $(P<0.0001)$ than nontreated OVA-obese mice. We observed an interaction between obesity, allergen sensitization, and treatment with NK1-R antagonist for metabolic and systemic biomarkers, and for allergen sensitization and bronchial inflammation, showing a synergy between these variables.

Conclusion \& Clinical Relevance: In an experimental model of obesity and asthma in mice, NK1-R blockade improved metabolic and systemic biomarkers, as well as allergen sensitization and bronchial inflammation. These positive effects support a common pathway in the obese-asthma phenotype and highlight SP as a target with potential clinical interest in the obese-asthma epidemics.
\end{abstract}

Epidemiological and clinical data show that obesity increases the relative risk of developing asthma (1) by 2 - to 3 -fold in obese adults and 1- to 2-fold in obese children $(2,3)$. Moreover, obesity is associated with a distinct asthma phenotype characterized by increased asthma severity, decreased response to standard medications, impaired glucocorticoid response, and the presence of comorbidities and metabolic/ immune imbalances related to obesity $(4,5)$.

A relationship between obesity and asthma has been explained by inflammatory or mechanical hypothesis (6).
Inflammatory mediators produced by adipose tissue may participate in pulmonary immune responses; the chronic low-grade inflammation of the obese may increase the susceptibility to airway obstruction (2). On the other hand, the obese breathe chronically at a lower functional residual capacity with smaller tidal volumes, which may lead to airway narrowing (7). Nevertheless, the precise mechanisms of the asthma-obesity relationship remain unclear (3).

Neurogenic inflammation is a possible link between obesity and asthma that has not been addressed so far. The 
tachykinin substance P (SP) is involved in neurogenic inflammation by acting through its selective receptor - neurokinin1 receptor (NK1-R). substance $\mathrm{P}$ has been reported to participate in the pathophysiology of asthma $(3,8,9)$. Substance $\mathrm{P}$ increases bronchoconstriction and airway hyper-responsiveness after allergen challenge (9), and the expression of its selective NK1-R is increased in the airways of asthmatic patients (8). The development of tachykinin receptor antagonists for the treatment of human airway diseases has had some advances as well as disappointing cases (10). However, the clinical interest of blocking tachykinin receptors for asthma management was recently reviewed. In a systematic review of randomized controlled trials evaluating the effect of tachykinin receptor antagonists on human allergic asthma, five studies showed a positive effect of tachykinin receptor antagonists on airway responsiveness and lung function of asthmatic patients (11). Differences observed among studies may be related to the usefulness of these antagonists in some specific phenotypes of allergic asthma rather than for all types of asthma.

Substance $\mathrm{P}$ has been also implicated in obesity as an orexigenic neuropeptide (12). Blockade of NK1-R in diet-induced obese and genetically obese (ob/ob) C57BL6/J mice resulted in decreased weight, decreased food intake, and improvement of serum glucose levels (12). In a previous study, we demonstrated that diet-induced obese Balb/c mice showed higher levels of serum SP than lean mice, and allergen-sensitized obese mice showed even higher levels of serum SP compared to allergen-sensitized lean mice and nonsensitized obese animals (13). We also demonstrated that obesity and asthma exerted a synergic effect on SP levels, as the joint effect of obesity and asthma on serum SP levels was superior to the sum of the effect of these two conditions (13). Our previous results raised the hypothesis of a pathophysiological role of SP in the obese-asthma phenotype.

We aimed to study the effect of a selective SP antagonist receptor on metabolic and systemic biomarkers related to obesity and on asthma and allergic inflammation, using a mouse model of diet-induced obesity with OVA-induced allergic sensitization and aerosol challenge.

\section{Methods}

\section{Animals and study design}

Balb/c mice aged 6-8 weeks old were used (Charles-River, USA) in four separate experiments $(n=6-8$ animals per group, matched by initial weight): two control groups, OVAlean $(23.7 \pm 0.8 \mathrm{~g})$, and OVA-obese $(23.3 \pm 0.5 \mathrm{~g})$, and two NK1-R antagonist-treated groups, OVA-lean+CJ $(23.3 \pm 1.0 \mathrm{~g})$ and OVA-obese $+\mathrm{CJ}(23.5 \pm 0.5 \mathrm{~g})$. All in vivo manipulations were handled by trained technicians and in agreement with the Portuguese Act 1005/92 (number 3, iii) and European Community guidelines (86/609/EEC) for the use of experimental animals. This study was approved by the Portuguese National Authority for Animal Health. Mice in the 'obese' groups were fed a high fat diet ( $45 \mathrm{kcal} \%$ fat, $4.73 \mathrm{kcal} / \mathrm{gm}$, Research Diets \#D 12451) for 17 weeks. 'Lean' mice received normal diet $(11 \mathrm{Kcal} \%$ fat w/corn starch, $3.48 \mathrm{kcal} / \mathrm{gm}$, Research Diets \#D12328) for the same period.

The allergen sensitization and challenge protocol was started after 12 weeks on high fat or normal diets. As previously described (14), animals were systemically sensitized by means of intraperitoneal injections of $10 \mu \mathrm{g}$ ovalbumin (OVA, \#LS003048; Worthington, Biochemical Corporation, Lakewood, NJ, USA) adsorbed to $1.5 \mathrm{mg}$ of aluminum hydroxide $\mathrm{Al}(\mathrm{OH})_{3}$ as adjuvant (AlumImject; Pierce, Rockford, IL, USA) on days 1, 14, and 21. Animals received two consecutive aerosol challenges of 1\% OVA (in phosphatebuffered saline, PBS) for a period of $20 \mathrm{~min}$ on days 26 and $27(2 \times$ challenge $)$. The challenge was repeated on days 33 and 34 , before killing the mice. One day after the last aerosol challenge, mice were treated with an intraperitoneal injection of $300 \mu \mathrm{g}$ of a selective NK1-R antagonist CJ 12255 (Pfizer Inc., Groton, CT, USA) or saline solution for 7 consecutive days.

Glucose levels were measured using standard glucose test strips (LifeScan, Inc., Milpitas, CA, USA) prior to killing. Cardiac blood was collected and serum separated and frozen at $-80^{\circ} \mathrm{C}$. Serum OVA-specific IgE level was measured using a commercial available ELISA kit (\#3010; Chondrex Inc., Redmond, WA, USA), and serum insulin, IL-6, and resistin levels were measured by a Milliplex ${ }^{\circledR}$ map kit (\#MADPK$71 \mathrm{~K}$; Millipore Corporation, Billerica, MA, USA), accordingly to the manufacturer instructions. Differential cell count (500 cells/slide) was performed in bronchoalveolar lavage fluid (BALF) (15). A blinded investigator used a previously described reproducible inflammation score to estimate the extent of peribronchial infiltrates in lung histology. Five randomly selected tissue sections per mouse were scored, and inflammation scores were expressed as a mean value per animal. Histological sections of visceral adipose tissue (VAT) were used, and five images per animal were obtained and analyzed using NIH ImageJ $1.34 \mathrm{e}$ software (http://imagej.nih. gov/ij/). The surface area of adipocytes was expressed as $\mu \mathrm{m}^{2}$.

\section{Statistical analysis}

Data are presented as mean \pm SEM. All tests were performed using Prisma 5.00 (Graphpad Software, San Diego, CA, USA) and PASW Statistics 18.0 (SPSS Inc., Chicago, IL, USA). Results were analyzed by protected analysis of variance (ANOVA) factorial with Bonferroni correction, Student's $t$-test, Mann-Whitney $U$-test (adipocyte areas), and one-way ANOvA followed by Tukey's test, after logarithmic transformation (cell counts in BALF). Interaction between obesity, asthma, and CJ treatment was analyzed by linear regression approach to ANCOVA. A $P$ value $<0.05$ was considered significant.

\section{Results}

Effect of NK1-R antagonism on metabolic and systemic biomarkers

We first study whether NK1-R antagonism affected weight gain, daily food and energy intakes, adipocyte size, and 
serum levels of insulin, IL-6, and resistin. These parameters were significantly different between groups (weight gain, $P<0.0001$; daily food intake, $P<0.0001$; energy intake, $P<0.0001$; adipocyte areas, $P<0.0001$; insulin, $P=0.0237$; IL-6, $P=0.0471$; and resistin, $P=0.0003$ ).

We observed that OVA-obese mice treated with CJ $(\mathrm{OVA}$-obese $+\mathrm{CJ})$ had lower weight (Fig. 1A: OVA-obese vs OVA-obese $+\mathrm{CJ}, \quad P=0.0002)$, reduced daily food intake (Fig. 1B: OVA-obese $v s \quad$ OVA-obese $+\mathrm{CJ}, \quad P=0.0021$ ), reduced daily energy intake (Fig. 1C: OVA-obese vs OVA-obese $+\mathrm{CJ}, P=0.0021$ ), lower adipocyte areas (Fig. 1D: OVA-obese vs OVA-obese+CJ, $P<0.0001$ ), lower serum glucose (Fig. 1E; OVA-obese vs OVA-obese+CJ, $P=0.040$ ), lower serum insulin (Fig. 1F: OVA-obese vs OVA-obese+CJ, $P=0.030$ ), lower serum IL-6 (Fig. 1G: OVA-obese $v s$ OVA-obese $+\mathrm{CJ}, \quad P=0.0022$ ), and lower serum resistin (Fig. 1H: OVA-obese vs OVA-obese+CJ, $P=0.0043$ ). NK1-R antagonism showed no effect on metabolic and systemic biomarkers in OVA-treated lean mice (Fig. 1A-H). These results showed that when metabolic impairments related to the obese state persist in allergen-sensitized and challenged mice, NK1-R antagonism had a positive effect on obesity-related biomarkers. Tissue macroscopic evaluation did not reveal any signs of adverse reaction against the drug. Intestinal inflammation was not observed either.

\section{Effect of NK1-R antagonism on allergen sensitization and bronchial inflammation}

Then, we studied whether NK1-R antagonism affected serum anti-OVA-specific IgE, peribronchial inflammatory infiltrates surrounding bronchi, and BALF cellularity. Anti-OVAspecific $\operatorname{IgE}(P=0.0093)$ and peribronchial score $(P<0.0001)$ were significantly different between the four groups.

Ovalbumin-obese+CJ mice showed lower serum anti-OVA IgE (Fig. 2A: OVA-obese vs OVA-obese+CJ, $P=0.035$ ), lower peribronchial inflammatory score in lung histology (Fig. 2B: OVA-obese $v s$ OVA-obese+CJ, $P<0.0001$ ), and lower eosinophils in BALF (Fig. 2C: OVA-obese vs OVAobese+CJ, $P=0.043$ ). Regarding other inflammatory cells in BALF, although no statistical significance was achieved, OVA-obese+CJ mice showed a tendency for lower cell counts (Fig. 2C). Peribronchial inflammatory score was the unique parameter affected by NK1-R antagonism in OVA-lean mice (Fig. 2B: OVA-lean $v s$ OVA-lean+CJ, $P=0.013$ ). These results showed that NK1-R antagonism had a positive effect on allergen sensitization and bronchial inflammation in allergen-sensitized and challenged obese mice. Inflammatory cells in BALF seemed also positively affected by the treatment in these animals.

\section{Interaction between obesity, allergen sensitization and challenge, and treatment with NK1-R antagonist}

An interaction between obesity, allergen sensitization and challenge, and treatment with NK1-R antagonism was seen for metabolic and systemic biomarkers (final weight, $R=0.924, \quad P<0.0001 ;$ daily energy intake, $R=0.996$,
$P<0.0001 ;$ daily food intake, $R=0.985, \quad P<0.0001$; adipocyte areas, $R=0.638, \quad P<0.0001$; serum glucose, $R=0.554, P<0.0001$; serum insulin, $R=0.605, P=0.004$; serum IL-6, $R=0.605, P=0.035$; serum resistin, $R=0.799$, $P<0.0001$ ) and for allergen sensitization and bronchial inflammation (serum anti-OVA $\operatorname{IgE}, R=0.678, P=0.013$; peribronchial inflammatory score, $R=0.892, \quad P<0.0001$; eosinophils in BALF, $R=0.460, P=0.007$; lymphocytes in BALF, $R=0.349, \quad P=0.006 ;$ monocytes in BALF, $R=0.431, \quad P=0.014 ;$ total cells in BALF, $R=0.419$, $P=0.004)$.

The observation that serum IL-6 levels, eosinophils in BALF, and bronchial inflammation were decreased in both sensitized lean mice and sensitized obese mice treated with NK1-R antagonist demonstrated that not all effects of this compound were related to weight loss. This indicates a synergistic effect between obesity, allergic sensitization, and treatment. This synergy was confirmed by the observed interaction between these variables for systemic and metabolic biomarkers, allergen sensitization, and bronchial inflammation (supplemental material). These results, along with the findings in sensitized lean mice, revealed that the treatment was more effective when both obesity and allergen sensitization were simultaneously present, due to a joint effect of both variables on evaluated parameters.

\section{Discussion}

To our knowledge, this is the first study to show that NK1$\mathrm{R}$ antagonism improves metabolic and systemic biomarkers, allergen sensitization, and bronchial inflammation in a mouse model of obesity and asthma. We also found an interaction between obesity, allergen sensitization, and treatment with NK1-R antagonist, obtained by using a statistical analysis that tests the relationship between these variables and evaluates whether their simultaneous influence on considered parameters is not additive. This interaction may explain the positive effect of treatment in sensitized obese mice and the absence of effect in sensitized lean mice for the majority of the analyzed biomarkers. Because no information was available regarding the effect of SP on visceral adipose tissue of allergen-sensitized obese mice, we studied whether NK1-R antagonism affected the adipocyte surface areas. Also, as SP has been associated with insulin resistance and increased serum levels of IL-6 levels (16), we analyzed the effect of NK1-R antagonism on serum levels of glucose, insulin, IL-6, and resistin. Obese and allergen-sensitized mice treated with NK1-R antagonist had reduced weight, reduced daily food intake, reduced daily energy intake, lower adipocyte areas, lower serum glucose, lower serum insulin, lower serum IL-6, lower serum resistin, lower serum anti-OVA IgE, and lower peribronchial inflammatory score in lung histology. In BALF, lower eosinophils and a tendency for lower lymphocytes, monocytes, and total cells were found after challenge in OVA-sensitized obese mice treated with antagonist. In our study, OVA-sensitized lean mice lost no weight during NK1-R antagonism treatment. The published literature on the effect of CJ 12255 on weight and appetite 

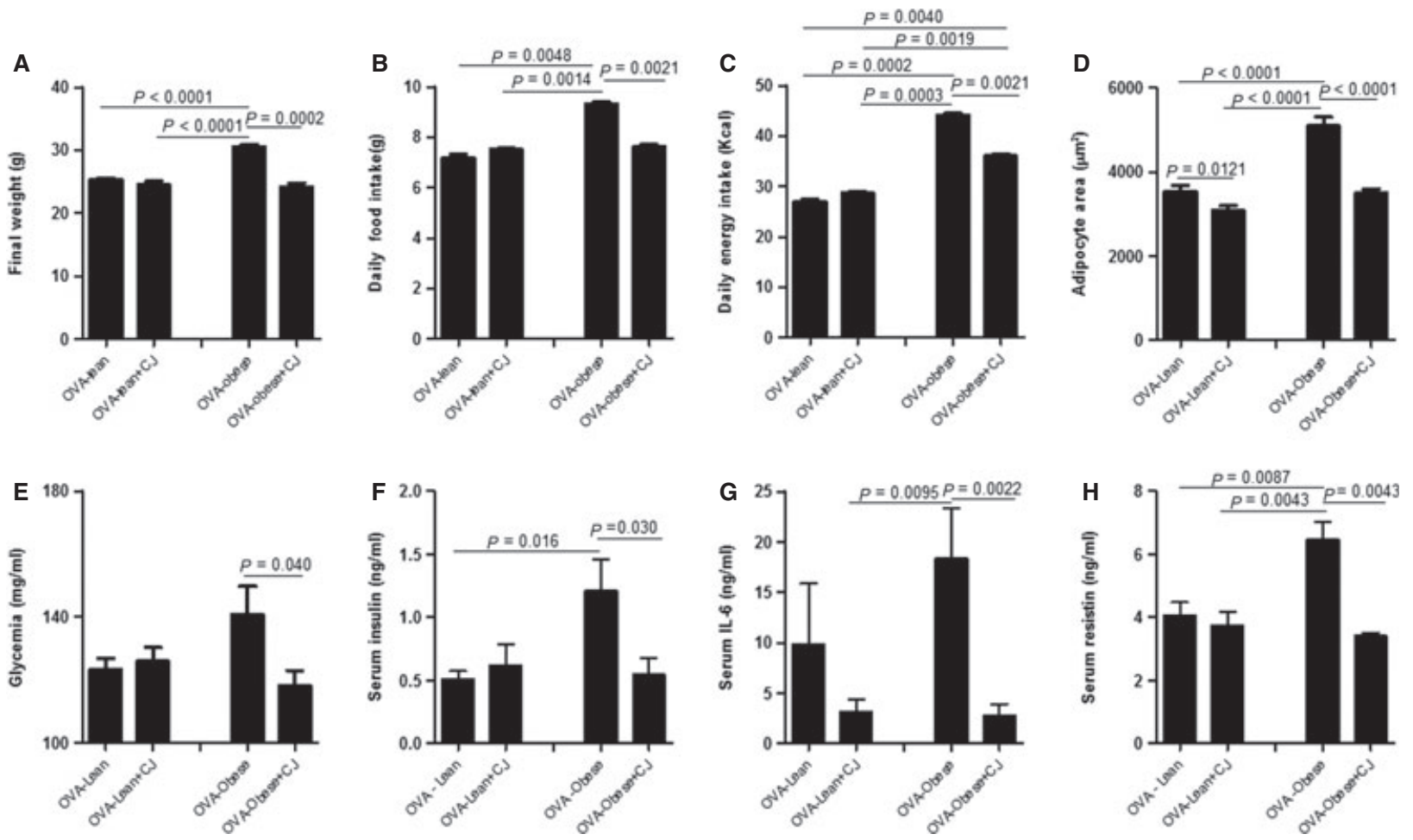

Figure 1 Effect of NK1-R antagonism (CJ) on metabolic and systemic biomarkers. OVA- treated obese mice (OVA-obese+CJ) showed lower final weight (A), reduced daily food intake (B),
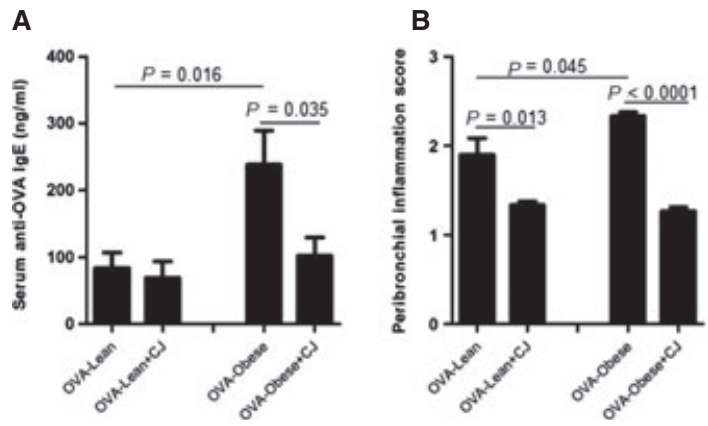

Figure 2 Effect of NK1-R antagonism on allergen sensitization and bronchial inflammation. OVA- treated obese mice (OVA-obese+CJ) showed reduced allergen sensitization (A) and lower inflammatory

showed that lean mice fed a high fat diet and treated with this NK-1R receptor had 3\% average (1-2 g) of weight loss (12). However, in that study, lean mice were fed a high fat diet during the CJ 12255 treatment. In our study, OVAsensitized lean mice maintained the normal diet during the experiment. This, along with the difference in the weights at reduced energy intake $(C)$, lower adipocyte size in visceral adipose tissue (D), reduced serum glucose $(E)$, reduced serum insulin (F), reduced serum IL-6 (G), and lower serum levels of resistin $(H)$.

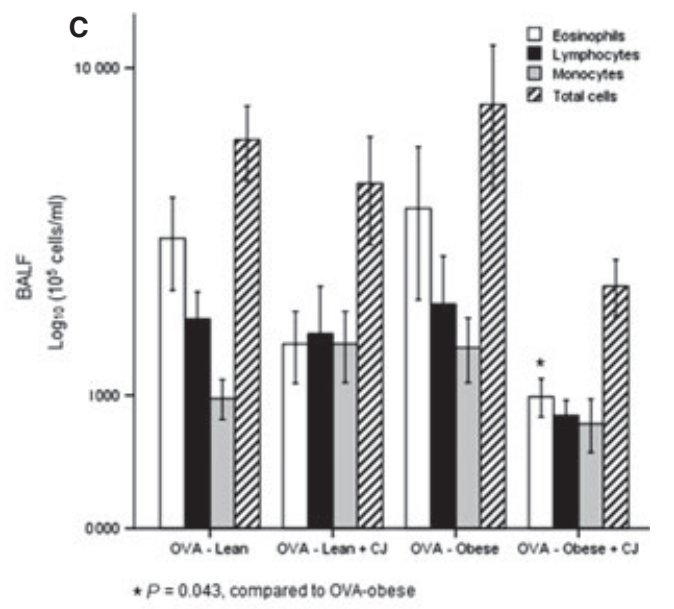

cell infiltrates surrounding bronchi (B). In bronchoalveolar lavage fluid (BALF), these animals showed reduced eosinophils and a tendency for lower inflammatory cell counts (C).

the beginning of the experiment with CJ 12255 between the published literature and ours (which are likely due to strainspecific characteristics), may also account for the differences observed.

The chronic low-grade inflammatory status of obesity is nowadays well established, reinforcing the role of obesity in 
the development and progression of allergic asthma (17). Accordingly, epidemiological studies have demonstrated a causality between obesity and asthma, with obesity preceding asthma development (18). In this association, excessive weight seems to be a risk factor for asthma. Eneli et al. (19), reviewed the effect of reversibility of this association, namely whether the reduction in body weight decreased the prevalence of asthma, asthma-related symptoms, or healthcare utilization. All 15 studies included in this systematic review, independently of the weight loss interventions, reported some improvement in asthma outcome (19). However, the heterogeneity of the studies and interventions to achieve weight loss called for the identification of an effective strategy for obese patients with asthma to reduce the medical, social, and economic impact associated with the obese-asthma phenotype.

In a scenario of neuroimmune interactions, tachykinins have been recognized as central neuropeptides (20). Tachykinin SP, neurokinin A (NKA) and neurokinin B (NKB), mediates their effects by stimulating their tachykinin receptors (NK1-R, NK2-R, and NK3-R receptors, respectively) (21). SP is the most extensively studied member of the tachykinin family and has been considered to be related to both asthma and obesity. In the airways of asthmatic patients, SP induces bronchoconstriction and airway hyperresponsiveness and increases mucus production and inflammatory cytokine release (22). Also, SP seems to be orexigenic and promoter of weight gain in mice (12) and related to insulin resistance and increased IL-6 levels in obese patients (16). Recently, we demonstrated that obesity and asthma in mice independently increase serum SP, and the presence of both pathologies further increases this level (13).

Because experimental data show that NK1-R is mainly involved in neurogenic inflammation, whereas NK2-R is considered relevant for airway smooth muscle contraction (21), and SP and NK1-R seem to be actively implicated in neurogenic inflammatory responses, we decided to use a NK1-R antagonist instead of an antagonist for NK2-R. A selective NK1-R antagonist (CJ 12,255) has already been tested in DIO and genetic $(o b / o b)$ mice models of obesity (12). Reduced food intake, body weight gain, glucose level, and insulin level were observed in both models of obesity, and these effects of NK1-R antagonist were considered to be associated with decreased appetite (12). In our study, we demonstrated the same positive effects on obesity-related metabolic parameters and, additionally, in allergen-related parameters. CJ 12 255, the NK1-R antagonist tested in our study, has been found to be anti-inflammatory in several conditions. It was demonstrated to reduce the pro-angiogenic factor $\mathrm{CCN} 1$ (23), the COX-2 induction, the SP-induced prostaglandin-E2 production, and to block SP-induced phosphorylation of JAK2, STAT3, and STAT5 in mice models of inflammatory diseases (24). In our study, we observed that both IL-6 and eosinophils were reduced by the treatment in sensitized obese mice, and a tendency for reduction in sensitized lean mice was also observed. It is recognized that the pro-inflammatory response to SP involves the activation of the nuclear factor (NF)-kB and the secretion of IL-6, IL-8, and tumor necrosis factor (TNF)-alpha (25) and that SP induces the recruitment of eosinophils in alveolar wall (26). Previous studies with selective NK1-R antagonists showed a reduction in eosinophils' recruitment (26) and reduction in the number of eosinophils in BALF (27). Because both studied models (obese-asthma in OVA-obese, and asthma in OVA-lean) represent inflammatory conditions, our results regarding the effect of tested NK1-R antagonist on both IL-6 levels and eosinophils' number are consistent with the literature and highlight the antiinflammatory effect of SP blockade.

The effect of NK1-R antagonism observed on serum glucose and insulin levels in our study is also in accordance with the literature (12). It was recently demonstrated that SP directly reduces insulin-mediated glucose uptake, providing evidence for an inhibitory role of SP in insulin signaling (28). It was demonstrated that mesenteric fat from NK1-R KO mice fed with HFD has reduced stress-activated protein kinase/c-Jun N-terminal kinase and protein kinase $\mathrm{C}$ activation compared with wild type (28). After glucose challenge, these authors observed that the glucose removal from the circulation was more efficient in NK1-R KO mice than in wild type and pair-fed controls (28). This suggested an NK1-Rmediated peripheral signaling on glucose metabolism.

In our study, we had further demonstrated an interaction between obesity, allergen sensitization and challenge, and treatment with NK1-R antagonism. Therefore, in our model, the effect of NK1-R on metabolic, systemic, and allergen inflammation biomarkers was not dependent on the lower weight gain of sensitized obese mice treated with antagonist, but to the synergistic effects of NK1-R blockade.

The molecular mechanisms implicated in the beneficial effect of SP antagonism in obese-asthma phenotype remain to be determined, but the role of SP in obese-asthma association may be separated from the role of this neuropeptide in overall inflammation. In our study, we provided some clues that SP is acting locally in both adipose tissue and the lungs, which are not characteristic of classical inflammation. The increased production of SP during the obese state $(13,16)$ and the up-regulation of NK1-R expression in preadipocytes (29) may indicate that SP is able to act locally in adipose tissue. In the present study, we demonstrated the effect of NK1-R on adipose tissue morphology by showing the reduction in adipocyte size, which may indicate that SP is acting directly in this tissue. In lungs, SP is able to induce the recruitment of eosinophil, neutrophil, and lymphocytes (30), and lung epithelium was demonstrated to overexpress NK1$\mathrm{R}$ in asthmatic patients (8). In our study, we demonstrated that peribronchial inflammation, evaluated by lung histology, is clearly reduced by the NK1-R antagonist, which may indicate that SP is also acting directly in the lungs. It is also known that NK1-R is expressed as a direct effect of SP presence in cell environment (31). We may speculate that increased SP levels produced during the obese state may induce the expression of NK1-R on adipose tissue and lungs in the obese-asthma phenotype, which clearly separate its role in obesity-associated aggravation of asthma from that in other inflammatory conditions. More insights into the molecular pathways involved are needed. 
We are aware that our experimental model induces allergen-specific $\operatorname{IgE}$ and $\operatorname{IgG1}$ as well as airway eosinophilia, which, in some degree, does not entirely mimic obeseasthma phenotype in humans. Nevertheless, the use of animal models to study the association between obesity and asthma has been shown to be of relevance (32), and environmental-related factors (allergens and diet) are specially relevant for the obesity and asthma epidemics seen in Western countries $(4,17)$. Moreover, experiments required to fully understand the cellular and molecular mechanisms must be performed in animals for ethical reasons, specially those testing pharmacological agents (33-35). However, when extrapolating data from mice to humans, care should be taken with anatomical differences in respiratory tract, types of adjuvants, chronicity of the disease, and age of animals (infants $v s$ adults).

In summary, our study provides evidence for the effectiveness of a NK1-R antagonism in reducing metabolic and allergic inflammatory parameters in obese-asthma phenotype in mice. Considering the role of neurogenic inflammation in the obese-asthma phenotype, SP may be an interesting candidate to target in the interplay between these two prevalent conditions that are a public health problem in the XXI century. Further studies are needed to confirm our results.

\section{Acknowledgments}

We kindly acknowledge Professor Tari Haahtela (Skin and Allergy Hospital, Helsinki University Central Hospital) and Dr. Luis Araújo for their critical review of the final manuscript. We acknowledge Pfizer Inc., USA, for gently providing the CJ 12255 . This study was partially funded by FCT (PTDC/SAU-OSM/102083/2008, PEst-OE/SAU/UI0038 /2011).

\section{Conflict of interest statement}

Nothing to declare.

\section{Authors contributions}

Renata Ramalho, André Moreira, and Raquel Soares participated in the design and conception of the study. Renata Ramalho performed laboratorial and statistical analysis and drafted the manuscript. Joana Almeida, Marília Beltrão, Ana Pirraco, Raquel Costa, Osksana Sokhatska, and Carmo Palmares performed and advised on methodological laboratorial analysis. Luísa Guardão headed the methodological handling of animal experiments. João T Guimarães, Luís Delgado, André Moreira, and Raquel Soares critically revised the manuscript for important intellectual content. All authors read and approved the final manuscript.

\section{Supporting Information}

Additional Supporting Information may be found in the online version of this article:

Data S1. Interaction between obesity, allergen sensitization, and treatment with NK1-R antagonist on analyzed parameters (linear regression approach to ANCOVA).

\section{References}

1. Beuther DA, Sutherland ER. Overweight, obesity, and incident asthma: a meta-analysis of prospective epidemiologic studies. Am J Respir Crit Care Med 2007;175:661-666.

2. Beuther DA. Recent insight into obesity and asthma. Curr Opin Pulm Med 2010;16:64-70.

3. Farah CS, Salome CM. Asthma and obesity: a known association but unknown mechanism. Respirology 2012;17:412-421.

4. Litonjua AA, Gold DR. Asthma and obesity: common early-life influences in the inception of disease. J Allergy Clin Immunol 2008;121:1075-1084.

5. Lugogo NL, Kraft M, Dixon AE. Does obesity produce a distinct asthma phenotype? J Appl Physiol 2010;108:729-734.

6. Shore SA. Obesity and asthma: possible mechanisms. J Allergy Clin Immunol 2008;121:1087-1093.

7. Shore SA. Obesity and asthma: cause for concern. Curr Opin Pharmacol 2006;6:230 236.

8. Chu HW, Kraft M, Krause JE, Rex MD, Martin RJ. Substance P and its receptor neurokinin 1 expression in asthmatic airways. J Allergy Clin Immunol 2000;106:713722 .
9. Hens G, Raap U, Vanoirbeek J, Meyts I, Callebaut I, Verbinnen B et al. Selective nasal allergen provocation induces substance P-mediated bronchial hyperresponsiveness. Am J Respir Cell Mol Biol 2011;44:517-523.

10. Farah CS, Kermode JA, Downie SR, Brown NJ, Hardaker KM, Berend $\mathrm{N}$ et al. Obesity is a determinant of asthma control, independent of inflammation and lung mechanics. Chest 2011;140:659-666.

11. Ramalho R, Soares R, Couto N, Moreira A. Tachykinin receptors antagonism for asthma: a systematic review. BMC Pulm Med 2011;11:41.

12. Karagiannides I, Torres D, Tseng Y, Bowe C, Carvalho E, Espinoza D et al. Substance $\mathrm{P}$ as a novel anti-obesity target. Gastroenterology 2008;134:747-755.

13. Ramalho R, Almeida J, Beltrão M, Pirraco A, Costa R, Sokhatska O et al. Neurogenic inflammation in allergen-challenged obese mice: a missing link in the obesity-asthma association? Exp Lung Res 2012;38: 316-324.

14. Dinh QT, Mingomataj E, Quarcoo D, Groneberg DA, Witt C, Klapp BF et al. Allergic airway inflammation induces tachykinin peptides expression in vagal sensory neurons innervating mouse airways. Clin Exp Allergy 2005;35:820-825.

15. Silveira PS, Palmares MC, Delgado L. [Bronchoalveolar lavage: methodology and clinical application] (translated from Portuguese). Rev Port Pneumol 1997;III:155178.

16. Fu J, Liu B, Liu P, Liu L, Li G, Wu B et al. Substance $\mathrm{P}$ is associated with the development of obesity, chronic inflammation and type 2 diabetes mellitus. Exp Clin Endocrinol Diabetes 2011;119:177-181.

17. Ma J, Xiao L, Knowles SB. Obesity, insulin resistance and the prevalence of atopy and asthma in US adults. Allergy 2010;65:14551463.

18. Ford ES. The epidemiology of obesity and asthma. J Allergy Clin Immunol 2005;115:897-909; quiz 910.

19. Eneli IU, Skybo T, Camargo CA Jr. Weight loss and asthma: a systematic review. Thorax 2008;63:671-676.

20. Veres TZ, Rochlitzer S, Braun A. The role of neuro-immune cross-talk in the regulation of inflammation and remodelling in asthma. Pharmacol Ther 2009;122:203-214. 
21. De Swert KO, Joos GF. Extending the understanding of sensory neuropeptides. Eur $J$ Pharmacol 2006;533:171-181.

22. Cheung D, van der Veen H, den Hartigh J, Dijkman JH, Sterk PJ. Effects of inhaled substance $\mathrm{P}$ on airway responsiveness to methacholine in asthmatic subjects in vivo. $J$ Appl Physiol 1994;77:1325-1332.

23. Koon HW, Zhao D, Xu H, Bowe C, Moss A, Moyer MP et al. Substance P-mediated expression of the pro-angiogenic factor CCN1 modulates the course of colitis. Am J Pathol 2008;173:400-410.

24. Koon HW, Zhao D, Zhan Y, Rhee SH, Moyer MP, Pothoulakis C. Substance P stimulates cyclooxygenase-2 and prostaglandin E2 expression through JAK-STAT activation in human colonic epithelial cells. J Immunol 2006;176:5050-5059.

25. Koon HW, Zhao D, Zhan Y, Simeonidis S, Moyer MP, Pothoulakis C. Substance Pstimulated interleukin-8 expression in human colonic epithelial cells involves protein kinase Cdelta activation. J Pharmacol Exp Ther 2005;314:1393-1400.

26. Tiberio IF, Leick-Maldonado EA, Miyahara L, Kasahara DI, Spilborghs GM, Martins
MA et al. Effects of neurokinins on airway and alveolar eosinophil recruitment. Exp Lung Res 2003;29:165-177.

27. Schuiling M, Zuidhof AB, Zaagsma J, Meurs H. Role of tachykinin NK1 and NK2 receptors in allergen-induced early and late asthmatic reactions, airway hyperresponsiveness, and airway inflammation in conscious, unrestrained guinea pigs. Clin Exp Allergy 1999;29(Suppl 2):48-52.

28. Karagiannides I, Stavrakis D, Bakirtzi K Kokkotou E, Pirtskhalava T, Nayeb-Hashemi $\mathrm{H}$ et al. Substance P (SP)-neurokinin-1 receptor (NK-1R) alters adipose tissue responses to high-fat diet and insulin action. Endocrinology 2011;152:2197-2205.

29. Karagiannides I, Kokkotou E, Tansky M, Tchkonia T, Giorgadze N, O'Brien M et al. Induction of colitis causes inflammatory responses in fat depots: evidence for substance $\mathrm{P}$ pathways in human mesenteric preadipocytes. Proc Natl Acad Sci U S A 2006;103:5207-5212.

30. Joachim RA, Sagach V, Quarcoo D, Dinh QT, Arck PC, Klapp BF. Neurokinin-1 receptor mediates stress-exacerbated allergic airway inflammation and airway hyperre- sponsiveness in mice. Psychosom Med 2004;66:564-571.

31. Quartara L, Maggi CA. The tachykinin NK1 receptor. Part I: ligands and mechanisms of cellular activation. Neuropeptides 1997;31:537-563.

32. Shore SA. Obesity and asthma: lessons from animal models. J Appl Physiol 2007;102:516528.

33. Krug N, Rabe KF. Animal models for human asthma: the perspective of a clinician. Curr Drug Targets 2008;9:438-442.

34. Sharma SK, Almeida FA, Kierstein S, Hortobagyi L, Lin T, Larkin A et al. Systemic FasL neutralization increases eosinophilic inflammation in a mouse model of asthma. Allergy 2012;67:328-335.

35. Zhou B, Ensell M, Zhou Y, Nair U, Glickstein J, Kermany $\mathrm{MH}$ et al. Prevention and treatment of DNA vaccine encoding cockroach allergen Bla g 1 in a mouse model of allergic airway inflammation. Allergy 2012;67:166-174. 